TRANSACTIONS OF THE

AMERICAN MATHEMATICAL SOCIETY

Volume 350, Number 10, October 1998, Pages 4149-4165

S $0002-9947(98) 02329-0$

\title{
ON FREE ACTIONS, MINIMAL FLOWS, AND A PROBLEM BY ELLIS
}

\author{
VLADIMIR G. PESTOV \\ Dedicated to my teacher, Professor Alexander V. Arhangel'skiu, on his 60th birthday
}

\begin{abstract}
We exhibit natural classes of Polish topological groups $G$ such that every continuous action of $G$ on a compact space has a fixed point, and observe that every group with this property provides a solution (in the negative) to a 1969 problem by Robert Ellis, as the Ellis semigroup $E(U)$ of the universal minimal $G$-flow $U$, being trivial, is not isomorphic with the greatest $G$-ambit. Further refining our construction, we obtain a Polish topological group $G$ acting freely on the universal minimal flow $U$ yet such that $\mathcal{S}(G)$ and $E(U)$ are not isomorphic. We also display Polish topological groups acting effectively but not freely on their universal minimal flows. In fact, we can produce examples of groups of all three types having any prescribed infinite weight. Our examples lead to dynamical conclusions for some groups of importance in analysis. For instance, both the full group of permutations $S(X)$ of an infinite set, equipped with the pointwise topology, and the unitary group $U(\mathcal{H})$ of an infinite-dimensional Hilbert space with the strong operator topology admit no free action on a compact space, and the circle $\mathbb{S}^{1}$ forms the universal minimal flow for the topological group Homeo $+\left(\mathbb{S}^{1}\right)$ of orientation-preserving homeomorphisms. It also follows that a closed subgroup of an amenable topological group need not be amenable.
\end{abstract}

\section{INTRODUCTION}

As was shown by Veech [38], every locally compact group admits a free action on a compact space. (Earlier Ellis proved this for discrete groups [7].) Little seems to be known to date about the existence of free actions on compacta for other kinds of topological groups. At the same time, this topic is linked to the following problem posed by Ellis in his 1969 Lectures on Topological Dynamics [8, Ch. 7] and readvertised by J. Auslander in the book [2, p. 120] and by de Vries in his recent monograph [40, Note IV.7.4.13]: is the canonical morphism from the greatest ambit (universal pointed compact $G$-flow) $\mathcal{S}(G)$ onto the Ellis semigroup $E(U)$ of the universal minimal flow $U=U(G)$ an isomorphism of ambits for a topological group $G$ ?

One obvious necessary condition for the answer to be positive is that $G$ act effectively on $U$, and a natural way to establish this is to prove that $G$ admits a

Received by the editors August 19, 1996.

1991 Mathematics Subject Classification. Primary 54H20.

Key words and phrases. Free actions on compacta, greatest ambit, universal minimal flow, Ellis semigroup, fixed point, effective actions, extremely amenable groups.

Research partially supported by the New Zealand Ministry of Research, Science and Technology through the project "Dynamics in Function Spaces" of the International Science Linkages Fund. 
free action on a compact space or, equivalently, acts freely on the greatest ambit. In this case, $G$ must act freely on the universal minimal flow, which is embedded into the greatest ambit. On the other hand, it is natural to ask if $G$ can behave in the 'opposite' way, that is, can it happen that every action of a topological group $G$ on a compact space has a fixed point?

This question appears in the 1970 paper of Mitchell [25]. The existence of a fixed point in every compact $G$-space is in fact an extremely strong version of amenability (cf. [11], Sec. II.4), which is why topological (semi)groups with this property have been called extremely amenable [16]. It appears that the first example of such a topological group was constructed in 1975 by Herer and Christensen [19] quite independently of the problematics of topological dynamics (in particular, the authors were unaware of either Mitchell's or Ellis's problems), and their paper, in turn, remains to date virtually unknown to topological dynamicists. At the same time, the existence of extremely amenable topological groups means that in general Ellis's problem is answered in the negative. Indeed, for any such group $G$ the enveloping semigroup of the universal minimal $G$-flow is trivial, $E(U)=\{e\}$, while the greatest ambit of every topological group $G$ contains a topological copy of $G$ and is therefore nontrivial if $G$ is. Apparently, this is the first time such an observation has been made.

While the example from [19] looks more like a genuine counter-example constructed with a particular purpose in mind, we exhibit vast classes of extremely amenable topological groups which are very natural.

5.4. Main Theorem. Let $G$ be a group of automorphisms of an infinite linearly ordered set $X$ acting transitively on $n$-subsets of $X$ for each $n$. Equip $G$ with the topology of pointwise convergence. Then every continuous action of the topological group $G$ on a compact space has a fixed point. Equivalently, the universal minimal $G$-flow is a singleton: $U(G) \cong\{*\}$.

Even if our examples were directly motivated by Ellis's problem, they appear to be of considerable interest on their own. Indeed, they lead to dynamical consequences for some topological groups having great prominence in analysis. According to the above result, the topological group Aut $(\mathbb{Q}, \leq)$ is extremely amenable. Among topological groups containing it as a topological subgroup are the full symmetric group $S(X)$ of an infinite set $X$ endowed with the topology of simple convergence, the unitary group $U(\mathcal{H})$ of an infinite-dimensional Hilbert space equipped with the strong operator topology, and the group of self-homeomorphisms of the Hilbert cube, Homeo $\left(I^{\omega}\right)$, with the compact-open topology. Our result means that none of these groups acts freely on any compact space. At the same time, the groups Homeo $\left(I^{\omega}\right)$ and $S(X)$ act effectively on their universal minimal flows. Such examples seem to be the first of their kind.

The property of extreme amenability is shared by the group Homeo $+(I)$ of all orientation-preserving homeomorphisms of the closed unit interval with the compact-open topology, as well as by the group $\mathrm{Homeo}_{+}(\mathbb{R})$. As another consequence, the universal minimal flow of the topological group Homeo $+\left(\mathbb{S}^{1}\right)$ is isomorphic to $\mathbb{S}^{1}$ itself. Such instances where a topological group lends itself to an explicit description of the universal minimal flow are uncommon (indeed, possibly this is the first such example ever). 
As yet another corollary of our major result, we observe that the class of topological groups acting freely on compacta is not closed under formation of topological quotient groups and therefore does not form a variety in the sense of [26].

Also, we show that the discrete free group $F_{2}$ on two generators is contained in Aut $(\mathbb{Q}, \leq)$ as a closed topological subgroup. Therefore, a closed subgroup of an amenable topological group in general need not be amenable, in sharp contrast to the well known result for locally compact groups. (Cf. e.g. [17], Th. 3.2.2.)

A natural question to ask in view of the existence of extremely amenable topological groups is whether or not the ambits $\mathcal{S}(G)$ and $E(U)$ are isomorphic when the action of $G$ on $\mathcal{S}(G)$ is free. We show that the answer to this is also negative: through refining the previous construction, we obtain an example of a Polish topological group acting freely on its greatest ambit yet such that $\mathcal{S}(G)$ and $E(U)$ are non-isomorphic. There are examples of such topological groups having any prescribed infinite weight.

After the present paper was circulated in August 1996 as a Victoria University of Wellington research report, the author learned from Eli Glasner that examples of extremely amenable topological groups had been known to him for quite a while, and also - again, independently - to H. Furstenberg and B. Weiss, but never published. Since then, Glasner's examples were written up and made public [13]. Those examples, which are different from but can be linked to that in [19], are of a totally different nature from ours and include even monothetic topological groups. Later on I was made aware of a recent solution by Glasner [12], [13] of Ellis's problem in the negative for the acting group $G=\mathbb{Z}$, which is of course the pre-eminent case.

For the most part, we restrict our attention to the special case where a topological group $G$ has a neighbourhood basis formed by open subgroups - or, equivalently, the greatest ambit $\mathcal{S}(G)$ is zero-dimensional. In passing, we single out a class of topological groups which are typically very far from being locally compact and yet act freely upon their greatest ambits: the so-called $P$-groups, characterized by the property that every $G_{\delta}$ subset is open [5]. This result is surprising, as $P$-groups have been used for producing 'nasty' counter-examples [30]-[32].

It is important to realize that the reported results (those by Herer and Christensen [19], Glasner [12], [13], and the present author) do not 'close' Ellis's problem but rather reshape it, giving substance to further open questions. We discuss them at the end of the article.

\section{Preliminaries and Conventions}

All topological groups in this article are assumed to be Hausdorff, all topological spaces Tychonoff (completely regular $T_{1}$ ), and all uniform spaces $(X, \mathcal{U})$ separated $\left(\cap \mathcal{U}=\Delta_{X}\right)$. The full symmetric group of a set $X$ is denoted by $S(X)$. Any action $\tau: G \rightarrow S(X)$ of a group $G$ on a set $X$ is interpreted as an action on the left, that is, we associate to it a map $G \times X \rightarrow X,(g, x) \mapsto g x \equiv \tau_{g} x$. By a $G$-flow we mean a compact $G$-space. The group Homeo $(X)$ of all homeomorphisms of a locally compact space $X$ onto itself is always equipped with the compact-open topology. For an orientable topological manifold $X$, Homeo $+(X)$ denotes the subgroup of orientation-preserving homeomorphisms.

Let $(X, \mathcal{U})$ be a uniform space. For a cover $\gamma$ of $X$, set $\tilde{\gamma}=\bigcup\{W \times W: W \in$ $\gamma\} \subseteq X^{2}$. We say that $\gamma$ is subordinated to $V \in \mathcal{U}$ if $\tilde{\gamma} \subseteq V$. Recall that $\gamma$ is 
uniform $\left[21\right.$, p. 199] if $\tilde{\gamma} \in \mathcal{U}$. We denote by $\mathcal{C}^{*} \mathcal{U}$ the finest totally bounded uniformity on $X$ contained in $\mathcal{U}$. It is the coarsest uniformity making each $f \in C^{*}(X, \mathcal{U})$ uniformly continuous, where $C^{*}(X, \mathcal{U})$ stands for the algebra of all bounded uniformly continuous real-valued functions on $(X, \mathcal{U})$. The completion of $\left(X, \mathcal{C}^{*} \mathcal{U}\right)$ is a compactum, called the Samuel compactification of $(X, \mathcal{U})$. In particular, the Stone$\breve{C e c h}$ compactification $\beta X$ of a topological space $X$ is the Samuel compactification of $\left(X, \mathcal{U}_{X}\right)$, where $\mathcal{U}_{X}$ is the finest compatible uniformity.

If $X$ is compact, then $\mathcal{U}_{X}$ is the unique compatible uniformity, and every finite open cover $\gamma$ is automatically uniform [21, Th. 6.33]. A compactum $X$ is zerodimensional if and only if a basis of $\mathcal{U}_{X}$ is formed by entourages of the form $\tilde{\gamma}$, where $\gamma$ are finite disjoint open covers of $X$.

Let $G$ be a topological group. For a subset $V \subseteq G$ we denote

$$
V_{\risingdotseq}=\left\{\left(x, y \in G^{2} \mid x y^{-1} \in V\right\}\right.
$$

and

$$
V_{\dashv}=\left\{\left(x, y \in G^{2} \mid x^{-1} y \in V\right\} .\right.
$$

(Here the subscripts $\gg$ and 7 conveniently indicate the position of the inversion

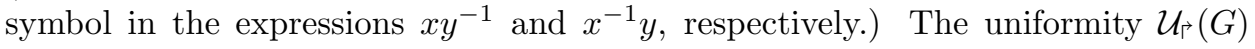
with a base formed by all sets $V_{\text {}}$, as $V$ runs over neighbourhoods of $e_{G}$, is called the left uniform structure by some authors [2], [8], [38], [39] and the right uniform structure by others [20], [21], [6]. We will denote by $C_{P}^{*}(G)$ the algebra formed by all bounded $\mathcal{U}_{\Gamma}$-uniformly continuous real-valued functions on $G$, that is, the bounded $f: G \rightarrow \mathbb{R}$ with

$$
\forall \epsilon>0, \exists V \ni e_{G}, \forall x, y \in G,\left(x y^{-1} \in V\right) \Rightarrow|f(x)-f(y)|<\epsilon .
$$

Every continuous action $\tau$ of a topological group $G$ on a uniform space $(X, \mathcal{U})$ by uniform homeomorphisms extends uniquely to an action of $G$ on the Samuel compactification $(\widehat{X}, \widehat{\mathcal{C} * \mathcal{U}})$. The extended action is continuous if $\tau$ is motion equicontinuous in the sense that for every $V \in \mathcal{U}$ the set $\{g \in G \mid \forall x \in X,(x, g x) \in V\}$ forms a neighbourhood of unity in $G$ [4, Th. 3.1]; [39]. (See also [23] for a more general result.)

An ambit is a compact $G$-space $X$ with a distinguished point $x \in X$ such that the orbit $G \cdot x$ is everywhere dense in $X$. Since $\mathcal{U}_{\vec{\Gamma}}(G)$ is motion equicontinuous with respect to the left action of $G$ on itself $\left(\tau_{g} h=g h\right)$, the Samuel compactification of $\left(G, \mathcal{U}_{\Gamma}(G)\right)$ forms an ambit with $e_{G}$ as the distinguished point. It is denoted by $\mathcal{S}(G)$ and called the greatest ambit for $G$. The choice of name is justified by the following universal property. For every $G$-ambit $(X, x)$ the orbit map $g \mapsto g x$ is $\mathcal{U}_{\vec{P}}$-uniformly continuous and therefore extends to a unique continuous map (onto) $\mathcal{S}(G) \rightarrow X$ sending $e_{G}$ to $x$ and commuting with the action of $G$ [35], [4], [2], [40].

The canonical action of a topological group $G$ on the greatest ambit $\mathcal{S}(G)$ is evidently effective. In fact, it enjoys a stronger property which would be best expressed by the unused adjective 'topologically effective.'

1.1. Proposition. (Teleman [35]; see also [4, Th. 3.2].) For any topological group $G$, the canonical action of $G$ on the greatest ambit $\mathcal{S}(G)$ is an isomorphism of $G$ with a topological subgroup of Homeo $\mathcal{S}(G)$.

By Zorn's Lemma, $\mathcal{S}(G)$ contains a minimal compact $G$-invariant subset, $U$, which forms a universal minimal $G$-flow in the sense that for every minimal $G$-flow 
$X$ there exists a continuous equivariant surjection $U \rightarrow X$. The universal minimal flow $U=U(G)$ is unique up to an isomorphism for any topological group $G$. (See $[8],[2],[40]$.

The Ellis, or enveloping, semigroup $E(X)$ of a compact $G$-space $X$ is the closure of the image of $G$ in $X^{X}$ in the pointwise topology. It forms a $G$-ambit with the distingushed point $\operatorname{Id}_{X}$ and the action of $G$ defined by $\tau_{g}(f)=g \circ f$ (loc. cit.). Ellis's problem which stimulated the present investigation is whether the canonical morphism $\mathcal{S}(G) \rightarrow E(U)$ is an isomorphism of ambits for a topological group $G$ [7], $[2],[40]$.

By $\widehat{G}$ we denote the completion of a topological group $G$ with respect to the twosided uniform structure [6, Sec. 2.1]. The following is a straightforward corollary of Teleman's result (1.1) and the well-known completeness of the group Homeo $(X)$ in the two-sided uniform structure.

1.2. Assertion. Let $G$ be a topological group. Then $\mathcal{S}(G)$ is canonically isomorphic with $\mathcal{S}(\widehat{G})$, and $U(G)$ is isomorphic with $U(\widehat{G})$.

\section{Generalia on free aCtions and fixed points}

2.1. Proposition. For a topological group $G$ and a subgroup $H<G$ the following are equivalent.

(i) $H$ has a fixed point in $\mathcal{S}(G)$;

(ii) $H$ has a fixed point in every compact $G$-space;

(iii) $H$ has a fixed point in every minimal compact $G$-space;

(iv) $H$ has a fixed point in the universal minimal compact $G$-space, $U(G)$;

(v) for every finite collection $g_{1}, g_{2}, \ldots, g_{d} \in H, d \in \mathbb{N}$, and every $V \in \mathcal{C}^{*} \mathcal{U}_{\vec{r}}(G)$ there is $a b \in G$ with $\left(b, g_{i} b\right) \in V$ for all $i=1,2, \ldots, d$.

Proof. As (iv) $\Leftrightarrow$ (iii) $\Leftrightarrow$ (ii) $\Leftrightarrow$ (i) $\Rightarrow$ (v) are obvious, only (v) $\Rightarrow$ (i) needs a proof. Assume (v) is satisfied and fix an arbitrary finite collection $g_{1}, g_{2}, \ldots, g_{d} \in H$. For each $V \in \mathcal{C}^{*} \mathcal{U}_{\Gamma}(G)$, choose an element $b_{V} \in G$ such that $\left(b_{V}, g_{i} b_{V}\right) \in V$ for all $i=1,2, \ldots, d$. Since $\mathcal{S}(G)$ is compact, the net $\left\{b_{V}: V \in \mathcal{C}^{*} \mathcal{U}_{\vec{\Gamma}}(G)\right\}$ has a cluster point [21, Th. 5.2], say $p \in \mathcal{S}(G)$. We claim that $p$ is a common fixed point for all $g_{i}$. Indeed, let $U$ be any neighbourhood of $p$ in $\mathcal{S}(G)$. Find a $V \in \mathcal{C}^{*} \mathcal{U}_{\overrightarrow{ }}(G)$ with $V^{2}[p] \subseteq U$. Since $g_{i}$ are (uniform) self-homeomorphisms of $\mathcal{S}(G)$, there exists a $W \in \mathcal{C}^{*} \mathcal{U}_{\vec{r}}(G)$ such that $W^{2} \subseteq V$ and $\left(g_{i} x, g_{i} y\right) \in V$ whenever $(x, y) \in W$. Since $p$ is a cluster point, there is a $W^{\prime} \in \mathcal{C}^{*} \mathcal{U}_{\vec{r}}(G)$ with $W^{\prime} \subseteq W$ and $b_{W^{\prime}} \in W[p]$. Now one has $\left(p, b_{W^{\prime}}\right) \in W,\left(b_{W^{\prime}}, g_{i} b_{W^{\prime}}\right) \in W^{\prime} \subseteq W$, and $\left(g_{i} b_{W^{\prime}}, g_{i} p\right) \in V$. Therefore, $\left(p, g_{i} p\right) \in W \circ W \circ V \subseteq V^{2}$ and $g_{i} p \in U$. Since $U$ is an arbitrary neighbourhood of $p$, we have established that every finite collection of points of $H$ has a common fixed point in $\mathcal{S}(G)$.

For any finite subset $K \subseteq H$ denote by $\Phi_{K}$ the set of all common fixed points for $K$ in $\mathcal{S}(G)$; it is closed and nonempty. The collection $\mathcal{F}=\left\{\Phi_{K}: K \in \mathcal{P}_{\omega}(H)\right\}$ forms a prefilter, because $\Phi_{K} \cap \Phi_{L}=\Phi_{K \cup L}$. Since $\mathcal{S}(G)$ is compact, $\cap \mathcal{F} \neq \emptyset$, and any element of this intersection is a common fixed point for all of $H$.

The two most important particular cases are those where $H=G$ and where $H$ is a cyclic subgroup generated by a $g \in G$. They lead to the following corollaries.

2.2. Corollary. For a topological group $G$ the following are equivalent. 
(i) $G$ has a fixed point in $\mathcal{S}(G)$;

(ii) $G$ has a fixed point in every compact $G$-space;

(iii) the universal minimal $G$-flow is trivial;

(iv) every minimal compact $G$-space is trivial;

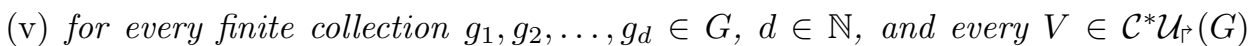
there is $a b \in G$ with $\left(b, g_{i} b\right) \in V$ for all $i=1,2, \ldots, d$.

2.3. Corollary. For a topological group $G$ the following are equivalent.

(i) $G$ acts freely on the greatest ambit, $\mathcal{S}(G)$;

(ii) $G$ admits a free action on a compact space;

(iii) $G$ acts freely on some minimal compact space;

(iv) $G$ acts freely on the universal minimal compact space $U(G)$;

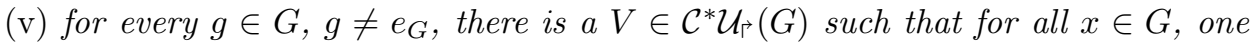
has $(x, g x) \notin V$.

Denote by $\mathcal{F C}$ the class of all topological groups admitting free actions on compact spaces. (Equivalently: $G \in \mathcal{F C}$ iff $G$ acts freely on $\mathcal{S}(G)$.) The main result of Veech [38] states that $\mathcal{F C}$ contains all locally compact groups. Here is a summary of some simple properties of the class $\mathcal{F C}$.

2.4. Proposition. (i) If a topological group $H$ admits a continuous monomorphism into a group $G \in \mathcal{F C}$, then $H \in \mathcal{F C}$. In particular, the class $\mathcal{F C}$ is closed under formation of topological subgroups.

(ii) The class $\mathcal{F C}$ is closed under the infinite direct products equipped with the Tychonoff topology.

(iii) If a topological group $G$ contains an open normal subgroup $H$ belonging to $\mathcal{F C}$, then $G \in \mathcal{F C}$.

Proof. (i) and (ii) are obvious. (iii) follows from two facts: (a) the discrete group $H \backslash G$ is in $\mathcal{F C}$; (b) the restriction of $\mathcal{C}^{*} \mathcal{U}_{\Gamma}(G)$ to $H$ coincides with $\mathcal{C}^{*} \mathcal{U}_{\risingdotseq}(H)$.

2.5. Corollary. Every maximally almost-periodic group is in $\mathcal{F C}$. In particular, if $X$ is a Tychonoff topological space, then the free topological group $F(X)$ (see [1], [14], [10], [20]) is in $\mathcal{F C}$.

Proof. Every free topological group is MAP, as was observed by Gelbaum [10]. (His note [10] is flawed, cf. [27], but not so far as this result is concerned.)

2.6. Corollary. The class $\mathcal{F C}$ is not closed under formation of topological factorgroups and therefore does not form a variety of topological groups in the sense of [26].

Proof. Every topological group $G$ is a factor-group of the free topological group $F(G)$ on the underlying space of $G$ [14]. At the same time, as will be shown in Section 5 - or as follows from the results of [19] $-\mathcal{F C}$ does not contain all topological groups.

\section{Greatest ambits having Dimension zero}

3.1. Assertion. Let $\mathcal{U}$ be a uniformity on a set $X$ having a basis of entourages consisting of equivalence relations on $X$. Let $V \in \mathcal{C}^{*} \mathcal{U}$. Then there are an equivalence relation $R \in \mathcal{U}$ and a finite disjoint cover $\gamma$ of $X$ by $R$-saturated subsets, subordinated to $V$. Moreover, $\tilde{\gamma} \in \mathcal{C}^{*} \mathcal{U}$. 
Proof. Since $\mathcal{C}^{*} \mathcal{U}$ is totally bounded, there is a finite $\mathcal{U}$-uniform open cover $\left\{W_{1}, \ldots, W_{k}\right\}$ of $X$, subordinated to $V$. For some equivalence relation $R \in \mathcal{U}$ and every $x \in X, R[x] \subseteq W_{i}$ for a suitable $i$. Set $U_{i}=\left\{x \in X: R[x] \subseteq W_{i}\right\}$; the collection $\left\{U_{1}, \ldots, U_{k}\right\}$ is an open cover of $X$, and for each $i, U_{i}=\pi_{R}^{-1} \pi_{R}\left(U_{i}\right)$, where $\pi_{R}: X \rightarrow X / R$ is the factor-map. Refine $\left\{\pi_{R} U_{1}, \ldots, \pi_{R} U_{k}\right\}$ to a disjoint cover $\left\{V_{1}^{\prime}, \ldots, V_{k}^{\prime}\right\}$ of the discrete uniform space $X / R$ and set $\gamma=\left\{\pi_{R}^{-1}\left(V_{i}^{\prime}\right): i=\right.$ $1, \ldots, k\}$. It remains to notice that the finite disjoint cover $\gamma$ of $X$ by $R$-saturated subsets is automatically $\mathcal{C}^{*} \mathcal{U}$-uniform, because $\tilde{\gamma}^{-1} \circ \tilde{\gamma}=\tilde{\gamma}$.

3.2. Corollary. Let $G$ be a topological group with a neighbourhood basis consisting of open subgroups. A basis for the uniformity $\mathcal{C}^{*} \mathcal{U}_{\vec{\Gamma}}(G)$ is formed by finite covers of the form $\left\{\pi_{H}^{-1}\left(V_{i}\right), i=1,2, \ldots, n\right\}$, where $H$ is an open subgroup of $G, \pi_{H}: G \rightarrow$ $H \backslash G$ is the factor map $(g \mapsto H g), n \in \mathbb{N}$, and $\left\{V_{i}: i=1,2, \ldots, n\right\}$ is a finite disjoint cover of the right factor-space $H \backslash G$.

Proof. Equivalence relations of the form $H_{\gg}$ form a basis for $\mathcal{U}_{\gg}(G)$, so Assertion 3.1 can be applied.

3.3. Corollary. Let $G$ be a topological group in which open subgroups form a neighbourhood basis. Then the greatest ambit of $G$ is canonically homeomorphic to the limit of the projective spectrum,

$$
\mathcal{S}(G)=\lim _{H} \beta(H \backslash G),
$$

taken over all open subgroups $H<G$.

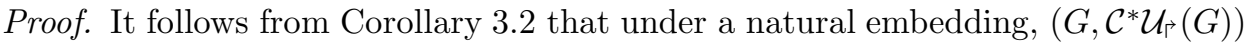
is a uniform subspace of the limit of the projective spectrum of uniform spaces,

$$
\varliminf_{\longleftarrow}\left(H \backslash G, \mathcal{C}^{*} \mathcal{U}_{H \backslash G}\right),
$$

where $\mathcal{U}_{H \backslash G}$ denotes the finest uniformity on the discrete factor-space.

3.4. Proposition. Let $G$ be a topological group. The greatest ambit $\mathcal{S}(G)$ is zerodimensional if and only if open subgroups form a neighbourhood basis in $G$.

Proof. $\Rightarrow$ : Assume $\operatorname{dim} \mathcal{S}(G)=0$. Then a basis of the unique uniform structure on $\mathcal{S}(G)$ is formed by equivalence relations of the form $\tilde{\gamma}$, where $\gamma$ runs over all finite disjoint open covers of $X$. For any such $\gamma$, the set

$$
\{g \in G: \forall x \in X,(x, g x) \in \tilde{\gamma}\}
$$

clearly is a subgroup of $G$, and such sets produce a neighbourhood basis for $G$ (Proposition 1.1).

$\Leftarrow$ follows from Corollary 3.3.

3.5. Remark. I was informed by Michael Megrelishvili and Scarr Tzvi of Bar-Ilan University (Israel) that Proposition 3.4 was known to them but never published.

\section{A NEW Class OF GROUPS ACTING FREELy ON COMPACTA}

Here we show that the class of topological groups admitting free actions on compact spaces extends beyond that formed by locally compact groups and their immediate derivatives (like MAP groups, subgroups of products, etc.).

A $P$-space is a topological space $X$ with the property that the intersection of every countable family of open subsets is open. A P-group is a topological group $G$ that is a $P$-space [5]. Evidently, only discrete $P$-groups are locally compact, and 
overall the class of $P$-groups is rather exotic. Yet from the dynamical viewpoint $P$-groups form a generalisation of discrete groups which is both natural and nontrivial. Indeed, since each $P$-group $G$ evidently has a neighbourhood basis formed by open subgroups, the greatest ambit of such a group is zero-dimensional (Proposition 3.4) and, as we prove below, the action of $G$ on $\mathcal{S}(G)$ is free. At the same time, $P$-groups need not have equivalent left and right uniformities [30], [31] and ipso facto can behave very much unlike discrete groups.

4.1. Examples. 1. If $G$ is any topological group, then the ultrapower $G_{\mathcal{U}}^{I}$ of $G$ formed with respect to an ultrafilter $\mathcal{U}$ on a set $I$ is the factor-group of $G^{I}$ by the equivalence relation

$$
x \stackrel{\mathcal{U}}{\sim} y \Leftrightarrow\left\{i \in I: x_{i}=y_{i}\right\} \in \mathcal{U}
$$

equipped with the factor-topology of the box product topology on $G^{I}$. If $\mathcal{U}$ is a $\delta$-incomplete free ultrafilter, then $G_{\mathcal{U}}^{I}$ is a $P$-group; it is non-discrete whenever $G$ is [3], [31].

2. If $K$ is an ordered field of an uncountable cofinality type and $n \in \mathbb{N}$, then $G L(K, n)$ forms a $P$-group with respect to the natural product topology coming from $K^{n^{2}}$, if $K$ is equipped with the order topology. The topologo-algebraic properties of such groups can be rather nontrivial [30]-[32].

3. There exist $P$-groups admitting no nontrivial continuous homomorphisms to locally compact groups. Let $X$ be an uncountable set. An alternating subgroup, $A(X)$, of the full symmetric group $S(X)$ consists of products of an even number of transpositions of pairs of elements of $X$. All subgroups of $A(X)$ of the form $S_{M}=\bigcap_{x \in M} \mathcal{S} t_{x}$, leaving each element of an arbitrary countable subset $M \subset X$ stable, form a basis for a non-discrete $P$-group topology on $A(X)$. Let $f$ be a non-trivial homomorphism from $A(X)$ to a locally compact group $G$. Since $A(X)$ is simple [6, p. 216], $f$ is a monomorphism. Since $A(X)$ contains a subgroup isomorphic to the group $S_{f i n}(X)$ of all finite permutations of $X$, it means the existence of a locally precompact group topology on $S_{f i n}(X)$, which is impossible in view of Gaughan's results [9]; [6], Th. 7.1.9 and Ex. 7.8.2.

Other examples and properties of $P$-groups can be found in [5].

4.2. Theorem. Every P-group acts freely on its greatest ambit.

Proof. Let $g \in G$. Fix an open subgroup $H^{\prime} \subseteq G$ with $H^{\prime} \not \nexists g$. The subgroup $H=\bigcap_{n \in \mathbb{Z}} g^{n} H^{\prime} g^{-n}$ is open in $G$ and $H \not \ngtr g$. Since for each $x \in G$ one has $g[x] \equiv$ $g(H x)=(g H) x=(H g) x=H(g x) \equiv[g x]$, the right factor-space $H \backslash G$ admits a natural left action of the cyclic subgroup $\langle g\rangle=\left\{g^{n}: n \in \mathbb{Z}\right\}$. Since any two different $\langle g\rangle$-orbits either coincide or are disjoint, one can choose a function $f: H \backslash G \rightarrow$ $\mathbb{Z}_{3}=\{0,1,2\}$ such that for each $[x] \in H \backslash G, f[x] \neq f(g[x])$, by constructing it for each orbit separately. (Observe that by the choice of $H, g[x]=[x]$ would imply $g=(g x) x^{-1} \in H$.) The function $\tilde{f}=\pi_{H} \circ f: G \rightarrow \mathbb{R}$, where $\pi_{H}: G \rightarrow H \backslash G$ is the factor-map, is constant on all right cosets $[x] \equiv H x$; therefore $x y^{-1} \in H$ implies $|f(x)-f(y)|=0$, which means that $f \in C_{\vec{P}}^{*}(G)$. For every $x \in G$ one has $|\tilde{f}(g x)-\tilde{f}(x)|=|f(g[x])-f[x]| \geq 1$. It remains to define $V \in \mathcal{C}^{*} \mathcal{U}_{\vec{\Gamma}}(G)$ by letting $(x, y) \in V \Leftrightarrow|\tilde{f}(x)-\tilde{f}(y)|<1$, and to apply Corollary 2.3.

4.3. Corollary. Every P-group acts freely on its universal minimal flow. 


\section{Groups haVing trivial Minimal FLOWS}

Let $X=(X, \leq)$ be a linearly ordered set. Denote by $\operatorname{Aut}(X)$ the group of all order automorphisms of $X$, equipped with the topology of pointwise (simple) convergence on $X$. A neighbourhood basis at unity consists of all open subgroups of the form

$$
\mathcal{S} t_{M}=\left\{g \in \operatorname{Aut}(X): \forall i=1, \ldots, n, g\left(m_{i}\right)=m_{i}\right\},
$$

where $M=\left\{m_{1}, m_{2}, \ldots, m_{n}\right\} \subset X$ is an arbitrary finite subset, $n \in \mathbb{N}$. This topology makes Aut $(X)$ into a topological group - in fact, a closed topological subgroup of the symmetric group $S(X)$ equipped with the pointwise topology [6, Sec.7.1]. Since the latter group is complete in its two-sided uniformity (loc. cit.), so is $\operatorname{Aut}(X)$.

Let us say that a group $G$ of automorphisms of a linearly ordered set $X$ is $\omega$ transitive if every finite subset of $X$ can be mapped onto any other subset of the same cardinality by an order automorphism $g \in G$. Call a linearly ordered set $X$ $\omega$-homogeneous if Aut $(X)$ acts $\omega$-transitively on $X$. Such sets exist in profusion, as the next simple result shows.

5.1. Assertion. Every linearly ordered skew field (division algebra) [29] is $\omega$ homogeneous as a linearly ordered set.

Proof. If $x_{1}<x_{2}<\cdots<x_{n}$ and $y_{1}<y_{2}<\cdots<y_{n}$ are two collections of elements of a linearly ordered skew field $F$, the following piecewise linear transformation of $F$ onto itself does the job:

$$
f(x)= \begin{cases}x+y_{1}-x_{1}, & \text { if } x \leq x_{1} \\ y_{i}+\frac{y_{i+1}-y_{i}}{x_{i+1}-x_{i}}\left(x-x_{i}\right), & \text { if } x_{i} \leq x \leq x_{i+1}, i=1,2, \ldots, n \\ x+y_{n}-x_{n}, & \text { if } x \geq x_{n}\end{cases}
$$

An example is the set $\mathbb{Q}$ of rational numbers with the usual order. Notice also that a group of automorphisms of an $\omega$-homogeneous linearly ordered set $X$ is $\omega$-transitive if and only if it is everywhere dense in Aut $(X)$.

5.2. Assertion. Let $G$ be an $\omega$-transitive group of automorphisms of an infinite linearly ordered set $X$. Let $M=\left\{m_{1}, m_{2}, \ldots, m_{n}\right\}$ be an arbitrary finite subset of $X$, where $n \in \mathbb{N}$. The right factor space $\mathcal{S} t_{M} \backslash G$ is naturally homeomorphic to the set $\mathcal{P}_{n}(X)$ of all $n$-subsets of $X$, equipped with the discrete topology. Namely, the mapping

$$
\mathcal{S} t_{M} \backslash G \ni[x] \equiv \mathcal{S} t_{M} x \stackrel{i_{n}}{\mapsto}\left\{x^{-1}\left(m_{1}\right), \ldots, x^{-1}\left(m_{n}\right)\right\} \in \mathcal{P}_{n}(X)
$$

is a correctly defined homeomorphism.

Proof. If $[x]=[y]$, then $y x^{-1} \in \mathcal{S} t_{M}$ and for all $i=1,2, \ldots, n$ one has $y x^{-1}\left(m_{i}\right)=$ $m_{i}$, which means $x^{-1}\left(m_{i}\right)=y^{-1}\left(m_{i}\right)$. Since $x$ is a bijection, $x^{-1}\left(m_{i}\right)$ and $x^{-1}\left(m_{j}\right)$ are different whenever $i \neq j$, and therefore $i_{n}$ is correctly defined. Assuming $i_{n}[x]=i_{n}[y]$, one deduces from $x$ and $y$ being order-preserving maps that $x^{-1}\left(m_{i}\right)=$ $y^{-1}\left(m_{i}\right)$ for all $i$, and therefore $y^{-1} x \in \mathcal{S} t_{M}$ and $[x]=[y]$. This means that $i_{n}$ is into. Now let $k_{1}<k_{2}<\cdots<k_{n}$ be elements of an arbitrary $n$-subset of $X$. By the finite transitivity of $G$, there exists an $x \in G$ with $x\left(k_{i}\right)=m_{i}$ for all $i=1,2, \ldots, n$. Hence $i_{n}$ is onto. Finally, $\mathcal{S} t_{M} \backslash G$ is discrete because $\mathcal{S} t_{M}$ is open in $G$. 
5.3. Lemma. Let $G$ be an $\omega$-transitive group of automorphisms of an infinite linearly ordered set $X$. Then for every finite collection $g_{1}, g_{2}, \ldots, g_{d} \in G$ and any

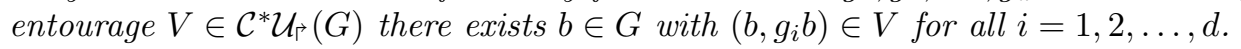

Proof. Find a disjoint finite cover $\gamma$, subordinated to $V$ and consisting of open subsets $V_{i} \equiv \mathcal{S} t_{M} \cdot V_{i}, i=1,2, \ldots, k$, where $M=\left\{m_{1}, m_{2}, \ldots, m_{n}\right\} \subseteq X$ (Corollary 3.2). Denote by $\gamma^{\prime}=\left\{V_{1}^{\prime}, V_{2}^{\prime}, \ldots, V_{k}^{\prime}\right\}$ the corresponding finite partition of $\mathcal{S} t_{M} \backslash G$. Identify $\mathcal{S} t_{M} \backslash G$ with $\mathcal{P}_{n}(X)$ as in 5.2. According to Infinite Ramsey's Theorem (see, e.g., [15, Th. 6.2.2]), there exists an infinite $B \subseteq X$ such that $\mathcal{P}_{n}(B) \subseteq V_{i}^{\prime}$ for some $i$.

Let $K=\left\{g_{i}^{-1}\left(m_{j}\right): i=1, \ldots, d, j=1, \ldots, n\right\} \cup M$. Write $K=\left\{\kappa_{1}, \ldots, \kappa_{t}\right\}$, where $\kappa_{1}<\kappa_{2}<\cdots<\kappa_{t}$. Since $B$ is infinite, there exist $\beta_{1}, \beta_{2}, \ldots, \beta_{t} \in B$ with $\beta_{1}<\beta_{2}<\cdots<\beta_{t}$. Due to the $\omega$-transitivity of $G$, there is an order automorphism $b \in G$ with $b\left(\beta_{1}\right)=\kappa_{1}, b\left(\beta_{2}\right)=\kappa_{2}, \ldots, b\left(\beta_{t}\right)=\kappa_{t}$.

The fact that $b^{-1}\left(m_{j}\right) \in B$ for all $j=1,2, \ldots, n$ means that $[b] \in \mathcal{P}_{n}(B) \subseteq V_{i}^{\prime}$ and therefore $b \in V_{i}$. Let $i=1,2, \ldots, d$ be arbitrary. One has $\left(g_{i} b\right)^{-1}\left(m_{j}\right)=$ $b^{-1} \circ g_{i}^{-1}\left(m_{j}\right) \in B$ for all $j=1,2, \ldots, n$ by the choice of $b$, and therefore $\left[g_{i} b\right] \in$ $\mathcal{P}_{n}(B) \subseteq V_{i}^{\prime}$ and $g_{i} b \in V_{i}$.

Since $\left\{V_{i}\right\}$ is subordinated to $V$, it follows that $\left(b, g_{i} b\right) \in V$, as required.

Lemma 5.3 and Proposition 2.2 imply the major result of our article.

5.4. Main Theorem. Let $G$ be an w-transitive group of automorphisms of an infinite linearly ordered set $X$, equipped with the pointwise topology. Then every continuous action of $G$ on a compact space has a fixed point. Equivalently, the universal minimal $G$-flow is a singleton: $U(G) \cong\{*\}$.

5.5. Corollary. For any infinite cardinal $\alpha$, there exists a topological group $G$ of weight $\alpha$ such that every continuous action of $G$ on a compact space has a fixed point.

Proof. It is well known that the purely transcendental extension of degree $\alpha$ of the field $\mathbb{Q}$ can be made into an ordered field [22]; denote it by $F$. It obviously has cardinality $\alpha$ and, according to Assertion 5.1, forms an $\omega$-homogeneous set. The group $\operatorname{Aut}(F)$ is easily verified to have weight $\alpha$.

The following in itself is not a new result, cf. [19], but the example appearing in it, which is entirely new, will be seen soon to have considerable interest on its own.

5.6. Corollary. There exists a non-trivial Polish (that is, separable completely metrizable) topological group $G$ such that every continuous action of $G$ on a compact space has a fixed point.

Proof. Such is Aut $(\mathbb{Q}, \leq)$.

5.7. Remark. It is well known that a topological group $G$ is amenable if and only if every affine action of $G$ on a compact convex set has a fixed point [2, th. 1.2.9]. A topological group is called strongly amenable if every proximal action of $G$ on a compact space has a fixed point [11, Sec. 2.3]. In view of this, we find it natural to adopt the terminology proposed by Granirer [16] and call a topological (semi)group $G$ extremely amenable if every action of $G$ on a compact space has a fixed point. 


\section{Dynamical consequences for some KnOWn Groups}

6.1. Assertion. Let $i: G \rightarrow H$ be a continuous monomorphism of topological groups with an everywhere dense image. If $G$ is extremely amenable, so is $H$.

Proof. The composition of the action $\tau: H \rightarrow$ Homeo $X$ with $i$ is an action of $G$ and therefore has a fixed point, whose stabiliser in $H$ contains the image of $G$ and thus coincides with $H$.

6.2. Theorem. Each of the following topological groups is extremely amenable:

(i) $\mathrm{Homeo}_{+}(I)$;

(ii) $\mathrm{HomeO}_{+}(\mathbb{R})$;

(iii) the stabilizer of any point $s \in \mathbb{S}^{1}$ in the group Homeo $+\left(\mathbb{S}^{1}\right)$.

Proof. Each of the three groups in question can be viewed as an $\omega$-transitive automorphism group of a linearly ordered set: $X=(0,1), \mathbb{R}$, and $\mathbb{S}^{1} \backslash\{s\}$ ordered counter-clockwise, respectively. By the Main Theorem 5.4, these groups are extremely amenable if equipped with the topology $\mathfrak{T}_{s}$ of simple convergence on the discrete set $X$. In each case it is easy to see that the compact-open topology is (strictly) coarser than $\mathfrak{T}_{s}$. Assertion 6.1 finishes the proof.

6.3. Theorem. None of the following topological groups acts freely on any compact space:

(i) the full symmetric group $S(X)$ of an infinite set $X$ with the topology of simple convergence,

(ii) the unitary group $U(\mathcal{H})$ of an infinite-dimensional Hilbert space with the strong operator topology,

(iii) the group of all self-homeomorphisms of the Hilbert cube, Homeo $\left(I^{\omega}\right)$, with the compact-open topology.

Proof. According to Proposition 2.4(i) and Theorem 5.4, a topological group $G$ admitting a continuous monomorphism from Aut $(\mathbb{Q})$ cannot act freely on a compact space. At the same time, one has

$$
\text { Aut }(\mathbb{Q})<S(\omega)<U\left(l_{2}\right)<\text { Homeo }\left(I^{\omega}\right),
$$

where $<$ denotes being a closed topological subgroup. (For the second inclusion see, e.g., [6, p. 263], and for the third [36].) Finally, $S(\omega)$ embeds into $S(X)$ as a closed topological subgroup, and similarly $U\left(l_{2}\right)$ embeds into $U(\mathcal{H})$.

6.4. Remark. The topological group Homeo $I^{\omega}$ acts effectively on its universal minimal flow, because it acts effectively on the minimal flow $I^{\omega}$. This gives us an example of a topological group whose action on its universal minimal flow is effective but not free.

Here is one more such result.

6.5. Theorem. The topological group $S(X)$ acts effectively on its universal minimal flow.

Proof. Let $\sigma$ be a transposition interchanging two fixed distinct elements $x_{1}$ and $x_{2}$ and leaving the rest of $X$ unchanged. Let $H=\mathcal{S} t_{x_{1}} \cap \mathcal{S} t_{x_{2}}$. The right factor-space $H \backslash S(X)$ can be naturally identified with the set $X^{(2)}$ of all ordered pairs of distinct elements of $X$, equipped with the discrete topology. The cyclic subgroup $\langle\sigma\rangle$ of order two, generated by $\sigma$, acts upon $H \backslash S(X) \cong X^{(2)}$ on the left by interchanging 
the coordinates $(\sigma(H x) \stackrel{\text { def }}{=} H \sigma(x))$. This action is obviously free. One can therefore construct a function $f: H \backslash S(X) \rightarrow \mathbb{Z}_{2}$ such that for all $[x] \in H \backslash S(X)$, one has $f[x] \neq f(\sigma[x])$. The composition $\tilde{f}$ of $f$ with the factor-map $S(X) \rightarrow H \backslash S(X)$ is $\mathcal{U}_{\Gamma}$-uniformly continuous. Therefore, $V=\left\{(x, y) \in S(X)^{2}:|\tilde{f}(x)-\tilde{f}(y)|<1\right\}$ is an element of $\mathcal{C}^{*} \mathcal{U}_{\Gamma}(S(X))$, and for each $x \in S(X),(x, \sigma x) \notin V$. Proposition 2.1 (the easy implication (iv) $\Rightarrow(\mathrm{v})$ ) means that $\sigma$ has no fixed points in the universal minimal flow of $S(X)$. Therefore, the kernel of inefficiency of the action of $S(X)$ on $U(S(X))$ does not contain $\sigma$. Since $S(X)$ has no non-trivial closed normal subgroups [6, Prop. 7.1.2(b)], this action is effective.

Here is another interesting example: the group Homeo $+\left(\mathbb{S}^{1}\right)$, even though obviously not amenable, lends itself to a complete description of the universal minimal flow.

6.6. Theorem. The universal minimal flow for the topological group Homeo $+\mathbb{S}^{1}$ is $\mathbb{S}^{1}$ itself.

Proof. Let $X$ be a minimal Homeo $+\mathbb{S}^{1}$-flow. According to Theorem 6.2 (iii), there is an $x \in X$ with $\mathcal{S} t_{s} \cdot x=x$. Therefore, the orbit map Homeo ${ }_{+} \mathbb{S}^{1} \rightarrow X, h \mapsto$ $h x$ factors through the left factor-space Homeo ${ }_{+} \mathbb{S}^{1} / \mathcal{S} t_{s}$, which is isomorphic as a Homeo $+\mathbb{S}^{1}$-flow to $\mathbb{S}^{1}$. The emerging continuous map $\mathbb{S}^{1} \rightarrow X$ is equivariant and onto.

6.7. Theorem. The Ellis problem is solved in the negative for the topological group Homeo $+\mathbb{S}^{1}$.

Proof. The Ellis semigroup $E\left(\mathbb{S}^{1}\right)$ is identified with the set of all orientation-preserving maps $\mathbb{S}^{1} \rightarrow \mathbb{S}^{1}$ (continuous or not), and the unique uniform structure on $E\left(\mathbb{S}^{1}\right)$ is that of pointwise convergence (induced from $\left(\mathbb{S}^{1}\right)^{1}$ ). We will accomplish our proof by constructing a $\mathcal{U}_{\Gamma}$-uniformly continuous function $f:$ Homeo $_{+} \mathbb{S}^{1} \rightarrow \mathbb{C}$ that is not uniformly continuous with respect to the pointwise uniformity. (Notice that Homeo $+\mathbb{S}^{1}$ canonically embeds into $E\left(\mathbb{S}^{1}\right)$ as a left Homeo $+\mathbb{S}^{1}$-flow.)

For each $\epsilon>0, \epsilon<1$, and any $t \in[0,1]$, denote by $l_{\epsilon}:[0,1] \rightarrow[0,1]$ the mapping sending 0 to $0, \epsilon$ to $1-\epsilon$ and 1 to 1 and whose restriction to each of the intervals $[0, \epsilon]$ and $[\epsilon, 1]$ is linear. Now set $h_{\epsilon}\left(e^{2 \pi \mathbf{i} t}\right)=e^{2 \pi \mathbf{i} l_{\epsilon}(t)}$. Clearly, $h_{\epsilon}$ is a correctly defined orientation-preserving homeomorphism of $\mathbb{S}^{1}$.

The uniformity $\mathcal{U}_{\overrightarrow{ }}\left(\right.$ Homeo $\left._{+} \mathbb{S}^{1}\right)$ is determined by the basic entourages of the form $\left\{(g, h): \forall s \in \mathbb{S}^{1},\left|g h^{-1}(s)-s\right|<\epsilon\right\}$ for $\epsilon>0$, or, equivalently, the entourages $V_{\epsilon}=\left\{(g, h): \forall s \in \mathbb{S}^{1},|h(s)-g(s)|<\epsilon\right\}$. (Here we identify $\mathbb{S}^{1}$ with the unit circle in $\mathbb{C}$.) For any $m, n \in \mathbb{N}, m<n$, the distance between $h_{2^{-n}}\left(e^{2 \pi \mathbf{i} 2^{-n}}\right)$ and $h_{2^{-n}}\left(e^{2 \pi \mathbf{i} 2^{-n}}\right)$ is easily seen to exceed $1 / 4$, which means that $\left(h_{2^{-n}}, h_{2^{-m}}\right) \notin V_{\frac{1}{4}}$. Therefore, the family $H=\left\{h_{2^{-n}}: n \in \mathbb{N}\right\}$ is right uniformly discrete in Homeo $+\mathbb{S}^{1}$. Since the latter group is separable and completely metrizable, any bounded right uniformly continuous function on $H$ extends to a similar function on Homeo $+\mathbb{S}^{1}$. Fix a bounded right uniformly continuous $f:$ Homeo $+\mathbb{S}^{1} \rightarrow \mathbb{C}$ with $f\left(h_{2^{-n}}\right)=$ $n \bmod 2 \in\{0,1\}$ for all $n \in \mathbb{N}$.

Now let $\left\{x_{1}, x_{2}, \ldots, x_{n}\right\}$ be any finite collection of elements of $\mathbb{S}^{1}$, and let $\epsilon>0$. Let $x_{i}=e^{2 \pi \mathbf{i} t_{i}}$ for all $i, 0 \leq t_{i}<1$. For any $m, n \geq \log _{2} \min \left\{t_{1}, t_{2}, \ldots, t_{n}, \epsilon\right\}$ one clearly has $\left|h_{n}\left(x_{i}\right)-h_{m}\left(x_{i}\right)\right|<\epsilon$ for all $i=1,2, \ldots, n$. At the same time, $\left|f\left(h_{m}\right)-f\left(h_{n}\right)\right|=1$ whenever $m$ and $n$ are of different parity. This means that 
$f$ is not uniformly continuous with respect to the uniform structure induced on Homeo $+\mathbb{S}^{1}$ from $\left(\mathbb{S}^{1}\right)^{\mathbb{S}^{1}}$, as required.

6.8. Corollary. There exists a Polish group $G$ acting 'topologically effectively' on its universal minimal flow $U$ (i.e., the action establishes an isomorphism of $G$ with a topological subgroup of Homeo $U$ ) yet such that the $G$-ambits $\mathcal{S}(G)$ and $E(U)$ are not isomorphic.

6.9. Remark. It may be worth recalling other remarkably odd dynamical properties of the same group: the Homeo $+\left(\mathbb{S}^{1}\right)$-flow $\mathbb{S}^{1}$ is not linearizable [23], [24], and the embedding of the stabilizer of any point $s \in \mathbb{S}^{1}$ into Homeo $+\left(\mathbb{S}^{1}\right)$ is an epimorphism of topological groups without dense range [37]. (A close link between two such kinds of properties is established in [33].)

\section{Groups $G$ aCting Freely on $\mathcal{S}(G)$ With $\mathcal{S}(G) \varsubsetneqq E(U)$}

7.1. Lemma. In an ordered skew field $X$ every interval of the form $(a, b),(-\infty, a)$, or $(b, \infty)$ is order-isomorphic to $X$.

Proof. The interval $(0,1)$ is order-isomorphic to $(1, \infty)$ via the map $x \mapsto$ $(1-x)^{-1}$ and to $(-\infty,-1)$ via $x \mapsto-x^{-1}$. Now the desired isomorphisms are patched together from the above maps composed with dilatations and translations.

The following was inspired by a similar result for the full permutation groups due to Shakhmatov [34].

7.2. Proposition. For every infinite cardinal $\alpha$, there exist a linearly ordered set $X$ of cardinality $\alpha$ and a free subgroup (of rank $\alpha$ ) of Aut $(X)$ acting $\omega$-transitively on $X$.

Proof. The free group $F(\alpha)$ of rank $\alpha$ is linearly orderable [28, Cor. 3.3] and therefore embeds into the multiplicative subgroup $S_{+}^{*}$ of positive elements of a linearly ordered skew field, $S$ [29, Th. 5.9]. Fix any such embedding and denote by $X$ the (underlying ordered set of) the miminal sub-skew-field of $S$ containing $F(\alpha)$. The group $F(\alpha)$ acts freely on $X$ by order automorphisms through the left multiplication, $F(\alpha) \ni a: x \mapsto a x \in X$. The cardinality of $X$ is $\alpha$, and so is the cardinality of the set $\mathcal{P}$ of all pairs $(A, B)$ of finite subsets of $X$ with Card $A=\operatorname{Card} B$. Establish a bijection $(A, B) \mapsto a_{(A, B)}$ between $\mathcal{P}$ and a free basis in $F(\alpha)$.

Let $(A, B) \in \mathcal{P}$ be arbitrary. Fix a $y_{(A, B)} \in X$ with $y_{(A, B)}>A \cup B$, and set $x_{(A, B)}=\max \left\{y_{(A, B)}, a_{(A, B)}^{-1} y_{(A, B)}\right\}+1$. Using Lemma 7.1 and the finite transitivity of $X$ (Proposition 6.1), one can construct piecewise an order isomorphism $\tau_{(A, B)}: X \rightarrow X$ such that

1. $\tau_{(A, B)}\left(-\infty, y_{(A, B)}\right)=\left(-\infty, y_{(A, B)}\right)$,

2. $\tau_{(A, B)}(A)=B$,

3. $\tau_{(A, B)}\left(y_{(A, B)}, x_{(A, B)}\right)=\left(y_{(A, B)}, a_{(A, B)} x_{(A, B)}\right)$,

4. if $x>x_{(A, B)}$, then $\tau_{(A, B)}(x)=a_{(A, B)} x$.

The resulting mapping $\tau: a_{(A, B)} \mapsto \tau_{(A, B)}$ from a free basis in $F(\alpha)$ to Aut $(X)$ lifts to a homomorphism $\widehat{\tau}: F(\alpha) \rightarrow \operatorname{Aut}(X)$. Clearly, the image of $F(\alpha)$ is everywhere dense in Aut $(X)$. Also, $\widehat{\tau}$ is one-to-one, because the image in Aut $(X)$ of any irreducible word $a=a_{\left(A_{1}, B_{1}\right)}^{\epsilon_{1}} a_{\left(A_{2}, B_{2}\right)}^{\epsilon_{2}} \ldots a_{\left(A_{n}, B_{n}\right)}^{\epsilon_{n}} \neq e$ acts non-trivially on any 
$x \in X$ with $x>\max \left\{x_{\left(A_{1}, B_{1}\right)}, x_{\left(A_{2}, B_{2}\right)}, \ldots, x_{\left(A_{n}, B_{n}\right)}\right\}$. Namely, a finite induction using the above condition (4) shows that $\widehat{\tau}(a)(x)=a x \neq x$, where $a$ is understood as an element of $F(\alpha)<X^{*}$ and the product is computed in $X$.

7.3. Theorem. Let $G$ be a group admitting an $\omega$-transitive effective action on a linearly ordered set. Denote by $\mathfrak{T}$ the topology of pointwise convergence corresponding to such an action. Let $G$ also support a profinite group topology, $\mathfrak{S}$. Then the topological group $G=(G, \mathfrak{T} \vee \mathfrak{S})$ acts freely on its greatest ambit $\mathcal{S}(G)$, yet the canonical morphism $\mathcal{S}(G) \rightarrow E(U)$ is not an isomorphism of $G$-ambits. Moreover, $\mathcal{S}(G)$ and $E(U)$ are not isomorphic as $G$-flows.

Proof. The greatest ambit of the precompact topological group $(G, \mathfrak{S})$ is isomorphic to the compact group $\widehat{G}^{\mathfrak{S}}$, the completion of $(G, \mathfrak{S})$ on which $G$ acts on the left, obviously freely; as a corollary, $(G, \mathfrak{T} \vee \mathfrak{S})$ acts freely upon its greatest ambit (Proposition 2.4.1). Denote by $\pi_{1}$ and $\pi_{2}$ the canonical morphisms from $\mathcal{S}(G, \mathfrak{T} \vee \mathfrak{S})$ onto $\mathcal{S}(G, \mathfrak{T})$ and onto $\mathcal{S}(G, \mathfrak{S}) \equiv \widehat{G}^{\mathfrak{S}}$, respectively. We aim to show that the product morphism $\pi_{1} \times \pi_{2}: \mathcal{S}(G, \mathfrak{T} \vee \mathfrak{S}) \rightarrow \mathcal{S}(G, \mathfrak{T}) \times \widehat{G}^{\mathfrak{S}}$ is one-to-one and therefore an embedding of $G$-ambits. This would mean that any minimal subset $U$ of $\mathcal{S}(G, \mathfrak{T} \vee \mathfrak{S})$ is contained in the product of $\widehat{G}^{\mathfrak{S}}$ and a minimal subset of $\mathcal{S}(G, \mathfrak{T})$, that is, a singleton; cf. Th. 5.4. Consequently, $U$ is isomorphic to $\widehat{G}^{\mathfrak{S}}$. Since the Ellis semigroup of the latter flow is isomorphic, as a $G$-ambit, to $\widehat{G}^{\mathfrak{S}}$ itself, it follows that $E(U)$ and $\mathcal{S}(G, \mathfrak{T} \vee \mathfrak{S})$ are non-isomorphic as $G$-flows. (Because, for example, the former flow is equicontinuous, while the latter is not, cf. [2, Ch. II, Prop. 1(ii) and Lemma 3].)

It suffices to show that for each $x \in \widehat{G}^{\mathfrak{S}}$, the restriction of $\pi_{1}$ to $\pi_{2}^{-1}(x)$ is one-toone. Let $y, z \in \pi_{2}^{-1}(x), y \neq z$. For some continuous $f: \mathcal{S}(G, \mathfrak{T} \vee \mathfrak{S}) \rightarrow \mathbb{Z}_{2}$ one has $f(y)=0, f(z)=1$. According to Corollary $3.2,\left.f\right|_{G}$ factors through some function $H \backslash G \rightarrow \mathbb{Z}_{2}$, where $H$ is a $\mathfrak{T} \vee \mathfrak{S}$-open subgroup of $G$; one can assume without loss in generality that $H=F_{1} \cap F_{2}$, where $F_{1}$ is $\mathfrak{T}$-open and $F_{2}$ is $\mathfrak{S}$-open in $G$. Since $\pi_{2}(y)=\pi_{2}(z)$, the elements $y$ and $z$ belong to the compact $\mathcal{S}(G, \mathfrak{T} \vee \mathfrak{S})$-closure of the same $F_{2}$-coset, say $F_{2} g$, of $G$.

We claim that $F_{2} g$ is $\mathfrak{T}$-everywhere dense in $G$, or equivalently, that so is $F_{2}$. Assuming it were not, the $\mathfrak{T}$-closure of $F_{2}$ would form a proper $\mathfrak{T}$-closed subgroup $D$ of finite index in $G$, leading to a nontrivial minimal left $(G, \mathfrak{T})$-flow $G / D$, in contradiction with Th. 5.4.

As a corollary, the restriction of the standard factor-map $\left(F_{1} \cap F_{2}\right) \backslash G \rightarrow F_{1} \backslash G$ to the image of $F_{2} g$ is a bijection onto $F_{1} \backslash G$, and $\left.f\right|_{F_{2} g}$ further factors through a function $F_{1} \backslash G \rightarrow \mathbb{Z}_{2}$ and is therefore $\left.\mathcal{C}^{*} \mathcal{U}_{\Gamma}(G, \mathfrak{T})\right|_{F_{2}}$-uniformly continuous. In view of this and because $F_{2} g$ is $\mathfrak{T}$-everywhere dense in $G$, the function $\left.f\right|_{F_{2} g}$ uniquely extends to a $\mathcal{C}^{*} \mathcal{U}_{\vec{\Gamma}}(G, \mathfrak{T})$-uniformly continuous function $\tilde{f}$ on $G$, and further to a continuous function $\bar{f}: \mathcal{S}(G, \mathfrak{T}) \rightarrow \mathbb{Z}_{2}$. It only remains to observe that $\bar{f}\left(\pi_{1}(y)\right)=0$, $\bar{f}\left(\pi_{1}(z)\right)=1$. Indeed, $\left.f\right|_{F_{2} g}=\left.\left(\bar{f} \circ \pi_{1}\right)\right|_{F_{2} g}$, and the values of any continuous function $f: \mathcal{S}(G, \mathfrak{T} \vee \mathfrak{S}) \rightarrow \mathbb{Z}_{2}$ at $y$ and $z$ are completely determined by the restriction of $f$ to $F_{2} g$.

7.4. Corollary. For every infinite cardinal $\alpha$, there exists a topological group $G$ of weight $\alpha$, acting freely on its greatest ambit $\mathcal{S}(G)$ (and therefore on the universal minimal flow $U(G)$ ) yet such that $\mathcal{S}(G)$ and $E(U)$ are not isomorphic as $G$-flows.

Proof. By Proposition 7.2, the free group $F(\alpha)$ of rank $\alpha$ admits an $\omega$-transitive effective action on a linearly ordered set of cardinality $\alpha$. It is well known that $F(\alpha)$ 
can be given a profinite group topology (cf. e.g. [27], Th. 1.4), and a standard machinery (see [1], Th. 2) enables one to extract from it a coarser profinite group topology of weight $\alpha$. Now Theorem 7.3 applies.

Applying Assertion 1.2 to Corollary 7.4 in the case $\alpha=\omega$, we obtain another major result of the present article.

7.5. Corollary. There exists a Polish group $G$ acting freely on its greatest ambit $\mathcal{S}(G)$ (and therefore on the universal minimal flow $U(G)$ ) yet such that $\mathcal{S}(G)$ and $E(U)$ are not isomorphic as $G$-flows.

7.6. Remark. The above examples exhibit yet another combination of dynamical properties: the action of $G$ on the universal minimal flow $U(G)$ is not 'topologically effective' even if it is free.

\section{Non-Amenable Closed Subgroups of AmenABle Groups}

Every closed subgroup of an amenable locally compact group is amenable ([17], Th. 2.3.2), and indeed this is one of the basic properties of amenability in the locally compact (in particular, discrete) setting. Here we show that for more general - in fact, even for Polish - topological groups this result is no longer true.

8.1. Theorem. The free group $F_{2}$ on two generators, equipped with the discrete topology, is isomorphic to a closed topological subgroup of Aut $(\mathbb{Q})$.

Proof. Choose $S$ and $X$ as in the first paragraph of the proof of Proposition 7.2, putting $\alpha=2$. Since the action of $F_{2}$ on $X$ by order automorphisms is free, the topology induced on this group from Aut $(\mathbb{Q})$ is discrete. (E.g., $\mathcal{S} t_{\{0\}} \cap F_{2}=\{e\}$, where 0 stands for the zero element of the skew field $S$.) Being a discrete subgroup, $F_{2}$ is automatically closed in Aut $(\mathbb{Q})$. The linearly ordered set $X$ is obviously a countable $\eta$-set, and therefore it is order isomorphic to $\mathbb{Q}$. (The classical reference for $\eta$-sets is [18].)

8.2. Corollary. A closed subgroup of an extremely amenable Polish topological group need not be amenable.

\section{Concluding Remarks}

The diversity of known examples of topological groups $G$ for which Ellis's problem is answered in the negative suggests the following.

Conjecture. Let $G$ be a topological group. The canonical morphism from the greatest ambit $\mathcal{S}(G)$ to the Ellis semigroup of the universal minimal $G$-flow $E(U(G))$ is an isomorphism if and only if $G$ is precompact.

Our examples substantiate the problem of describing in intrinsic terms those topological groups $G$ whose actions on universal minimal flows are effective or, better still, 'topologically effective.' The following question by Glasner [13] is of profound interest: does there exist a minimally almost periodic non-extremely amenable monothetic group? We do not know whether $U\left(l_{2}\right)$ (with the strong operator topology) acts effectively on its universal minimal flow. Theorem 6.6 tends to suggest that the Hilbert cube $I^{\omega}$ might serve as the universal minimal flow for the group Homeo $\left(I^{\omega}\right)$. In view of Corollary 8.2, we ask: is every topological group isomorphic with a subgroup of an (extremely) amenable topological group? 


\section{ACKNOWLEDGEMENTS}

I owe a debt of gratitude to Michael Megrelishvili and to Vladimir Uspenskij, from whom I learned a great deal in this area of knowledge. I benefitted from discussions with Joe Auslander (whom I thank for hospitality extended in October 1996 at the University of Maryland), Eli Glasner, and Alexander Kechris (who brought my attention to the reference [19]). Finally, I dedicate this article to my teacher Alexander V. Arhangel'skiu, who in 1983 suggested that I move to topological dynamics; my only regret is that it took me so long to heed his advice.

\section{REFERENCES}

1. A. V. Arhangel'skiur, Relations among invariants of topological groups and their subspaces, Russian Math. Surveys 35 (1980), no. 3, 1-23. MR 82e:54005

2. J. Auslander, Minimal Flows and Their Extensions, North-Holland Mathematics Studies 153, North-Holland, Amsterdam, 1988. MR 89m:54050

3. P. Bankston, Ultraproducts in topology, Gen. Topol. Appl. 7 (1977), 283-308. MR 56:16554

4. R. B. Brook, A constructions of the greatest ambit, Math. Systems Theory 4 (1970), 243-248. MR 42:1940

5. W. W. Comfort and K. Ross, Pseudocompactness and uniform continuity in topological groups, Pacific J. Math. 16 (1966), 483-496. MR 34:7699

6. D. Dikranjan, I. Prodanov, and L. Stoyanov, Topological Groups. Characters, Dualities, and Minimal Group Topologies, Monographs and Textbooks in Pure and Applied Mathematics 130, Marcel Dekker, Inc., NY-Basel, 1989. MR 91e:22001

7. R. Ellis, Universal minimal sets, Proc. Amer. Math. Soc. 11 (1960), 540-543. MR 22:8491

8. L Lectures on Topological Dynamics, Mathematical Lecture Note Series, W.A. Benjamin Inc., New York, 1969. MR 42:2463

9. E. D. Gaughan, Topological group structures of infinite symmetric groups, Proc. Nat. Acad. Sci. USA 58 (1967), 907-910. MR 35:6775

10. B. R. Gelbaum, Free topological groups, Proc. Amer. Math. Soc. 12 (1961), 737-743. MR 25:4025

11. S. Glasner, Proximal Flows, Lecture Notes in Math. 517, Springer-Verlag, Berlin-HeidelbergNY, 1976. MR 57:13890

12. __ Structure theory as a tool in topological dynamics, Lectures given during the Descriptive Set Theory and Ergodic Theory Joint Workshop (Luminy, June 1996), Tel Aviv University preprint, $26 \mathrm{pp}$.

13. _ On minimal actions of Polish groups, Tel Aviv University preprint, October 1996, 6 pp.

14. M. I. Graev, Free topological groups, Amer. Math. Soc. Transl. 35 (1951), 61 pp. MR 12:391c

15. R. L. Graham, B. L. Rothschild, and J. H. Spencer, Ramsey Theory, Wiley-Interscience, NY, 1980. MR 82b:05001

16. E. Granirer, Extremely amenable semigroups 2, Math. Scand. 20 (1967), 93-113. MR 35:3422

17. F. P. Greenleaf, Invariant Means on Topological Groups, Van Nostrand Mathematical Studies 16, Van Nostrand - Reinhold Co., New York, 1969. MR 40:4776

18. F. Hausdorff, Grundzüge der Mengenlehre, Leipzig, 1914. MR 11:88d (reprint)

19. W. Herer and J.P.R. Christensen, On the existence of pathological submeasures and the construction of exotic topological groups, Math. Ann. 213 (1975), 203-210. MR 54:495

20. E. Hewitt and K.A. Ross, Abstract Harmonic Analysis. Vol. 1 (2nd ed.), Springer-Verlag, New York, 1979. MR 81k:43001

21. J. L. Kelley, General Topology, D. van Nostrand, Inc., Princeton, NJ, 1955. MR 16:1136c

22. E. Makai, Jr., Notes on real closed fields, Ann. Univ. Sci. Budapest Eötvös Sect. Math. 13 (1970), 35-55. MR 46:9014

23. M. Megrelishvili, Compactification and factorization in the category of G-spaces, Categorical Topology (J. Adamek and S. MacLane, eds.), World Sci. Publ., Singapore, 1989, pp. 220-237. MR 92c:54031

24. _ Free topological G-groups, New Zealand J. Math. 25 (1996), 59-72. MR 97f:54047

25. T. Mitchell, Topological semigroups and fixed points, Illinois J. Math. 14 (1970), 630-641. MR 42:5245 
26. S. A. Morris, Varieties of topological groups, Bull. Austral. Math. Soc. 1 (1969), 145-160. MR 41:3655a

27. - Varieties of topological groups III, ibid. 2 (1970), 165-178. MR 41:3655c

28. B. H. Neumann, On ordered groups, Amer. J. Math. 71 (1949), 1-18. MR 10:428a

29. , On ordered division rings, Trans. Amer. Math. Soc. 66 (1949), 202-252. MR 11:311f

30. V. G. Pestov, Embeddings and condensations of topological groups, Math. Notes 31 (1982), 228-230. MR 83k:54004

31. Topological groups and algebraic envelopes of topological spaces, Ph.D. thesis, Moscow State University, Moscow, 1983, 78 pp. (in Russian).

32. , A test of balance of a locally compact group, Ukr. Math. J. 40 (1988), 109-111. MR 89e:22006

33. E Eimorphisms of topological groups by way of topological dynamics, New Zealand J. Math. 26 (1997), 257-262. CMP 98:07

34. D. B. Shakhmatov, Character and pseudocharacter in minimal topological groups, Math. Notes 38 (1985), 1003-1006. MR 87j:22004

35. S. Teleman, Sur la représentation linéaire des groupes topologiques, Ann. Sci. Ecole Norm. Sup. 74 (1957), 319-339. MR 20:3927

36. V. V. Uspenskiǔ, A universal topological group with countable base, Funct. Anal. Appl. 20 (1986), 160-161. MR 87i:22010

37. , The epimorphism problem for Hausdorff topological groups, Topology Appl. $\mathbf{5 7}$ (1994), 287-294. MR 95i:54046

38. W. A. Veech, Topological dynamics, Bull. Amer. Math. Soc. 83 (1977), 775-830. MR 57:7558

39. J. de Vries, On the existence of $G$-compactifications, Bull. Acad. Pol. Sci. Ser. Math. 26 (1978), 275-280. MR 58:31002

40. _ Elements of topological dynamics, Mathematics and Its Applications 257, Kluwer Academic Publ., Dordrecht-Boston-London, 1993. MR 94m:54098

School of Mathematical and Computing Sciences, Victoria University of Wellington, P.O. Box 600, Wellington, New Zealand

E-mail address: pestovv@member.ams.org 